\title{
Mobility of a two-dimensional electron gas in the AIGaAs/GaAs heterostructure: simulation and analysis
}

\author{
Vladislav Volcheck ${ }^{*}$ and Viktor Stempitsky \\ Belarusian State University of Informatics and Radioelectronics, Department \\ of Micro- and Nanoelectronics, 220013 Minsk, Belarus
}

\begin{abstract}
At temperatures above $100 \mathrm{~K}$, a two-dimensional electron gas generated at the $\mathrm{AlGaAs} / \mathrm{GaAs}$ heterointerface can be characterized by the three dominant scattering mechanisms: acoustic deformation potential, polar acoustic phonon and polar optical phonon. An analytical model describing the two-dimensional electron gas mobility controlled by these scattering processes as a function of the electron concentration and the temperature was developed and integrated into a device simulator package using a built-in $\mathrm{C}$ language interpreter. The electrical characteristics of a simple $\mathrm{AlGaAs} / \mathrm{GaAs}$ high electron mobility transistor were simulated using either the derived or a conventional bulk mobility model and the results were compared.
\end{abstract}

\section{Introduction}

One of the most important properties of semiconductor heterojunction structures is the mobility enhancement behavior first observed by Dingle et al. [1] in modulation-doped $\mathrm{AlGaAs} / \mathrm{GaAs}$ superlattices. A classic selectively-doped structure is comprised of AlGaAs doped with silicon and semi-insulating GaAs layers. Due to the electron affinity difference between the two materials, a triangular quantum well is formed where the energy levels for motion in the direction perpendicular to the heterojunction plane are quantized. For thin wells and temperatures not too high, only the lowest level is occupied. Since all electrons remain free to move in the other two directions, a two-dimensional electron gas is generated. The mobility enhancement behavior is attributed to the spatial separation between the electrons in GaAs and the donors in AlGaAs by a thin spacer layer, which decreases ionized impurity scattering. The field-effect control of the two-dimensional electron gas in modulation-doped structures underlies the operation of the high electron mobility transistor (HEMT) [2].

Analyzing III/V semiconductors and devices with position-dependent band structures is complicated by the lack of a thorough approach to modeling, as the development of III/V materials has routinely trailed the advanced silicon technology. The availability of accurate mobility models is indispensable to the reliable electrical characterization of a HEMT. However, popular device simulator packages for advanced materials, such as Blaze, include

*Corresponding author: vlad.volchek@bsuir.by 
low- and high-field mobility models only applicable to semiconductor bulk, but not to a two-dimensional electron gas. In this work, we develop and integrate into device simulation software using a built-in C language interpreter an analytical low-field model for the twodimensional electron gas mobility controlled by the acoustic deformation potential, the polar acoustic phonon and the polar optical phonon scattering processes.

\section{Simulation details}

The low temperature Hall mobility in doped bulk GaAs is limited by the ionized impurity scattering mechanism and is proportional to $\mathrm{T}^{3 / 2}$. At $77 \mathrm{~K}$, the mobility of bulk material with low compensation ratio can reach $1.5 \times 10^{5} \mathrm{~cm}^{2} /(\mathrm{V} \cdot \mathrm{s})$ for a donor density of $10^{14} \mathrm{~cm}^{-3}$ and reduce to $8000 \mathrm{~cm}^{2} /(\mathrm{V} \cdot \mathrm{s})$ for a donor density of $10^{17} \mathrm{~cm}^{-3}$ typical for GaAs field-effect transistors [3]. In a practical HEMT, the background donor concentration is approximately $10^{14} \mathrm{~cm}^{-3}$, but the large density of the two-dimensional electron gas $\left(\sim 10^{12} \mathrm{~cm}^{-2}\right)$ leads to screening of the Coulomb potential, which also enhances the mobility. The values as high as $1.95 \times 10^{5} \mathrm{~cm}^{2} /(\mathrm{V} \cdot \mathrm{s})$ have been achieved at $77 \mathrm{~K}[4]$.

At temperatures above $100 \mathrm{~K}$, the dominant scattering mechanism in the twodimensional electron gas formed at the $\mathrm{AlGaAs} / \mathrm{GaAs}$ heterointerface is polar optical phonon scattering [5]. Although contributing less to the two-dimensional electron gas mobility in relative terms, scattering by the acoustic deformation potential and polar acoustic phonon (piezoelectric) scattering should not be neglected [6]. As it was noted previously, ionized impurity scattering is very low due to the spacer layer and consequently is not accounted for in the mobility model.

\subsection{Acoustic deformation potential scattering}

The momentum relaxation time for acoustic deformation potential scattering is calculated as follows [6]:

$$
\tau_{\mathrm{ADP}}=\frac{\hbar^{3} v_{1}^{2} \rho w}{q^{2} m \kappa T \varphi_{\mathrm{AD}}^{2}},
$$

where $\hbar$ is the reduced Planck constant, $v_{1}$ is the velocity of the longitudinal acoustic phonon, $\rho$ is the density, $q$ is the elementary charge, $m$ is the electron effective mass, $\kappa$ is the Boltzmann constant, $T$ is the temperature and $\varphi_{\mathrm{AD}}$ is the acoustic deformation potential.

In (1), $w$ is the effective width of the two-dimensional electron gas, which is defined as the double average distance of the electronic wave function from the heterointerface into GaAs for the $0^{\text {th }}$ subband $z_{0}$ :

$$
w=2 z_{0}=5.5 \times 10^{-9}\left(\frac{10^{16}}{n}\right)^{\frac{1}{3}}
$$

where $n$ is the sheet electron concentration.

\subsection{Polar acoustic phonon scattering}

The momentum relaxation time for polar acoustic phonon (piezoelectric) scattering mechanism is calculated as follows [7]: 


$$
\tau_{\mathrm{PE}}=\frac{\pi \varphi_{\mathrm{AD}}^{2} q_{\mathrm{F}}}{h_{14}^{2} w}\left(\frac{9}{32}+\frac{13}{32}\left(\frac{v_{1}}{v_{\mathrm{t}}}\right)^{2} \frac{\Gamma_{\mathrm{t}}}{\Gamma_{1}}\right)^{-1} \tau_{\mathrm{ADP}},
$$

where $h_{14}$ is the piezoelectric constant and $v_{\mathrm{t}}$ is the velocity of the transverse acoustic phonon.

In (3), $q_{\mathrm{F}}$ is the wave vector on the Fermi surface determined by

$$
q_{\mathrm{F}}=\sqrt{2 \pi n},
$$

The factors $\Gamma_{1}$ and $\Gamma_{\mathrm{t}}$ are defined by

$$
\begin{gathered}
\Gamma_{1}=\sqrt{\left(\frac{4 \gamma_{1}}{3 \pi}\right)^{2}+1}, \\
\Gamma_{\mathrm{t}}=\sqrt{\left(\frac{4 \gamma_{\mathrm{t}}}{3 \pi}\right)^{2}+1},
\end{gathered}
$$

where

$$
\begin{aligned}
& \gamma_{1}=\frac{2 \hbar v_{1} q_{\mathrm{F}}}{\kappa T}, \\
& \gamma_{\mathrm{t}}=\frac{2 \hbar v_{\mathrm{t}} q_{\mathrm{F}}}{\kappa T} .
\end{aligned}
$$

\subsection{Polar optical phonon scattering}

Strictly speaking, a momentum relaxation time for polar optical phonon scattering cannot be determined. However, the following formula can be used as an approximation [8]:

$$
\tau_{\mathrm{POP}}=\frac{4 \hbar^{2} \pi \kappa_{\varepsilon} \varepsilon_{0}\left(1-5 \frac{V_{\mathrm{T}}}{E_{\mathrm{g}}}\right)}{q^{2} \sqrt{2 q m E_{\mathrm{POP}}\left(1+\frac{E_{\mathrm{POP}}}{E_{\mathrm{g}}}\right)} N_{\mathrm{P}}},
$$

where $\varepsilon_{0}$ is the dielectric constant, $V_{\mathrm{T}}$ is the thermal voltage, $E_{\mathrm{g}}$ is the band gap and $E_{\mathrm{POP}}$ is the polar optical phonon energy.

In (9), $\kappa_{\varepsilon}$ is the coupling constant:

$$
\frac{1}{\kappa_{\varepsilon}}=\frac{1}{\varepsilon_{\infty}}-\frac{1}{\varepsilon},
$$

where $\varepsilon_{\infty}$ is the high-frequency relative permittivity and $\varepsilon$ is the low frequency relative permittivity.

The temperature dependence of the energy gap is taken into account and is fitted to the empirical Varshni form [9]: 


$$
E_{\mathrm{g}}(T)=E_{\mathrm{g}}(0 \mathrm{~K})-\frac{\alpha T^{2}}{T+\beta},
$$

where $\alpha$ and $\beta$ are adjustable parameters.

The parameter $N_{\mathrm{p}}$ is the phonon Planck function:

$$
N_{\mathrm{P}}=\sqrt{\exp \left(\frac{E_{\mathrm{POP}}}{V_{\mathrm{T}}}\right)-1} .
$$

\subsection{Two-dimensional electron gas mobility model}

The relation between the $i$-mobility limited by one scattering mechanism and the corresponding $i$-momentum relaxation time is

$$
\mu_{\mathrm{i}}=\frac{q}{m} \tau_{\mathrm{i}}
$$

The combined mobility of the two-dimensional electron gas generated at the $\mathrm{AlGaAs} / \mathrm{GaAs}$ heterointerface and controlled by the three scattering mechanisms is approximated using Matthiessen's rule:

$$
\frac{1}{\mu_{2 \mathrm{DEG}}}(T, n)=\frac{1}{\mu_{\mathrm{ADP}}}+\frac{1}{\mu_{\mathrm{PE}}}+\frac{1}{\mu_{\mathrm{POP}}} .
$$

The values of the GaAs material parameters used during the simulations are given in Table 1 .

Table 1. GaAs material parameters.

\begin{tabular}{|l|c|c|c|}
\hline Parameter & Values & Units & References \\
\hline $\mathrm{m}$ & 0.067 & - & {$[10]$} \\
\hline$\rho$ & 5316 & $\mathrm{~kg} / \mathrm{m}^{3}$ & {$[11]$} \\
\hline $\mathrm{h}_{14}$ & $1.45 \times 10^{9}$ & $\mathrm{~V} / \mathrm{m}$ & {$[11]$} \\
\hline$v_{1}$ & 5137 & $\mathrm{~m} / \mathrm{s}$ & {$[12]$} \\
\hline$v_{\mathrm{t}}$ & 3024 & $\mathrm{~m} / \mathrm{s}$ & {$[12]$} \\
\hline$\varphi_{\mathrm{AD}}$ & 7 & $\mathrm{eV}$ & {$[13]$} \\
\hline$\varepsilon$ & 12.72 & - & {$[14]$} \\
\hline$\varepsilon_{\infty}$ & 10.9 & - & {$[14]$} \\
\hline$E_{\mathrm{POP}}$ & 0.03536 & $\mathrm{eV}$ & {$[13]$} \\
\hline
\end{tabular}

\subsection{Sotoodeh bulk material mobility model}

The low-field mobility in bulk GaAs can be described by a model of the Caughey-Thomas type that accounts for the donor density $N_{\mathrm{d}}$ and temperature, according to Sotoodeh et al. [15]:

$$
\mu_{0}\left(T, N_{\mathrm{d}}\right)=500+\frac{9400\left(\frac{T}{300}\right)^{-2.1}-500}{1+\left(\frac{T}{300}\right)^{-1.182}\left(\frac{N_{\mathrm{d}}}{6 \times 10^{16}}\right)^{0.394}} .
$$




\section{Results}

The temperature dependences of the two-dimensional electron gas mobility limited by each of the three scattering mechanisms and the combined mobility are presented in Figures 1 and 2 for the electron concentrations of $1 \times 10^{12} \mathrm{~cm}^{-2}$ and $2 \times 10^{12} \mathrm{~cm}^{-2}$, respectively.

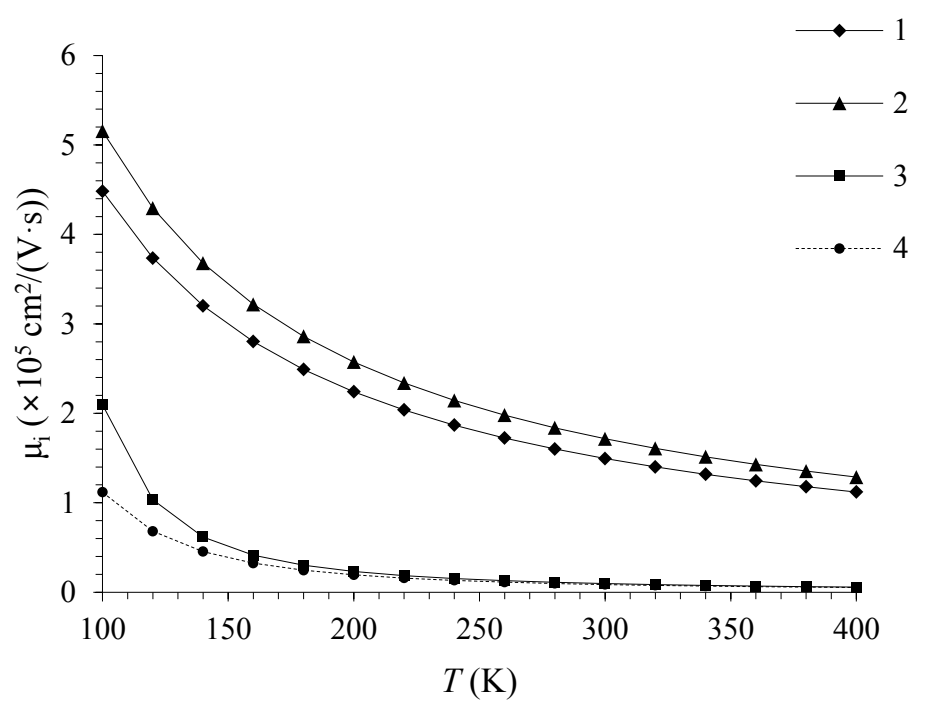

Fig. 1. Temperature dependence of the two-dimensional electron gas for $n=1 \times 10^{12} \mathrm{~cm}^{-2}: 1$ - acoustic deformation potential scattering, 2 - polar acoustic phonon scattering, 3 - polar optical phonon scattering, 4 - combined mobility.

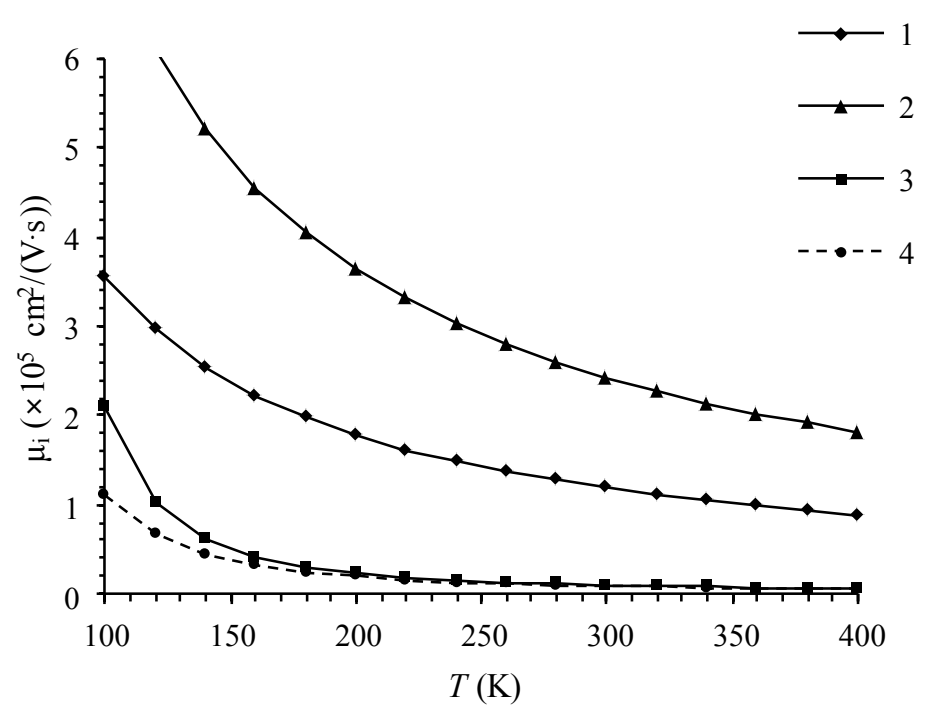

Fig. 2. Temperature dependence of the two-dimensional electron gas for $n=2 \times 10^{12} \mathrm{~cm}^{-2}: 1-$ acoustic deformation potential scattering, 2 - polar acoustic phonon scattering, 3 - polar optical phonon scattering, 4 - combined mobility.

As can be seen from (9), the polar optical phonon scattering rate is independent of the density of the two-dimensional electron gas. At the same time, the mobility controlled 
either by the acoustic deformation potential or the polar acoustic phonon scattering processes is very sensitive to the electron concentration. When $n=1 \times 10^{12} \mathrm{~cm}^{-2}$ at $300 \mathrm{~K}$ $\mu_{\mathrm{ADP}}$ equals to $1.494 \times 10^{5} \mathrm{~cm}^{2} /(\mathrm{V} \cdot \mathrm{s})$ and its contribution to the overall mobility is $5.754 \%$. In the second case, $\mu_{\mathrm{ADP}}$ reaches the value of $1.186 \times 10^{5} \mathrm{~cm}^{2} /(\mathrm{V} \cdot \mathrm{s})$ and its percentage increases to $7.248 \%$. The mobility controlled by polar acoustic phonon scattering behaves in a different way. If $n=1 \times 10^{12} \mathrm{~cm}^{-2}$ at $300 \mathrm{~K}, \mu_{\mathrm{PE}}$ equals to $1.715 \times 10^{5} \mathrm{~cm}^{2} /(\mathrm{V} \cdot \mathrm{s})$, making up $5.012 \%$ of the combined mobility. However, if $n=2 \times 10^{12} \mathrm{~cm}^{-2}$, it reaches the value of $2.426 \times 10^{5} \mathrm{~cm}^{2} /(\mathrm{V} \cdot \mathrm{s})$ and its contribution is only $3.542 \%$.

The two-dimensional electron gas mobility limited by polar optical phonon scattering and the overall mobility as functions of the electron concentration at $300 \mathrm{~K}$ are presented in Figure 3.

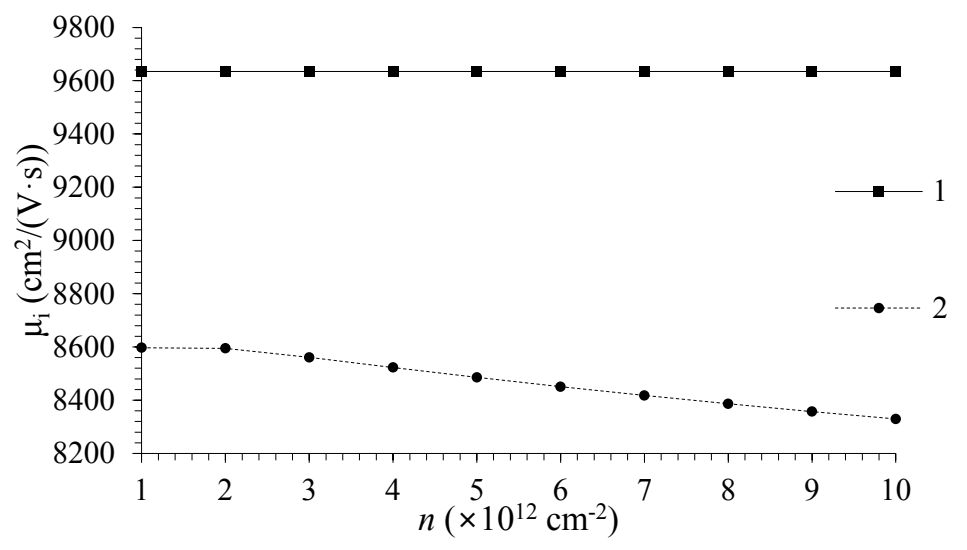

Fig. 3. Mobility of the two-dimensional electron gas as a function of its density at $300 \mathrm{~K}: 1$ - polar optical phonon scattering, 2 - combined mobility.

As shown in the chart, when the density of the two-dimensional electron gas is raised from the value of $2 \times 10^{12} \mathrm{~cm}^{-2}$ to $1 \times 10^{13} \mathrm{~cm}^{-2}$ the combined mobility decreases almost linearly from the value of $8595 \mathrm{~cm}^{2} /(\mathrm{V} \cdot \mathrm{s})$ to $8330 \mathrm{~cm}^{2} /(\mathrm{V} \cdot \mathrm{s})$. The contribution of the polar optical phonon scattering mechanism reduces by $3.087 \%$ due to the increasing impact of acoustic deformation potential scattering.

The principal difference between the developed two-dimensional electron gas mobility model and the Sotoodeh bulk material model is that the first accounts for the electron concentration, which is determined largely by the electron affinity difference and the donor impurity density in AlGaAs if the GaAs layer is moderately doped $\left(N_{\mathrm{d}} \sim 10^{14} \mathrm{~cm}^{-3}\right)$. The second model does not take into account the electron concentration and provides a spatially uniform mobility profile when the donor density and the temperature are constant.

In order to compare the two mobility models, a simplified AlGaAs/GaAs HEMT is simulated at $300 \mathrm{~K}$ using a self-consistent coupled Schrödinger-Poisson model that gives a quantized description of the density of states in the presence of quantum mechanical confining potential variations. The device structure is comprised of a $1.5 \mu \mathrm{m} \mathrm{GaAs}$ buffer layer $n$-type doped to a concentration of $10^{14} \mathrm{~cm}^{-3}$ and a $20 \mathrm{~nm} \mathrm{Al}_{0.3} \mathrm{Ga}_{0.7} \mathrm{As}$ barrier layer with a donor density of $10^{19} \mathrm{~cm}^{-3}$. The source-to-gate and the gate-to-drain distances equal to 1 and $3.5 \mu \mathrm{m}$, respectively. The length and the width of the gate are 0.5 and $150 \mu \mathrm{m}$, respectively. In Figure 4, the dependences of the drain current and the transconductance on the gate voltage at the drain voltage of $1 \mathrm{~V}$ are provided. 


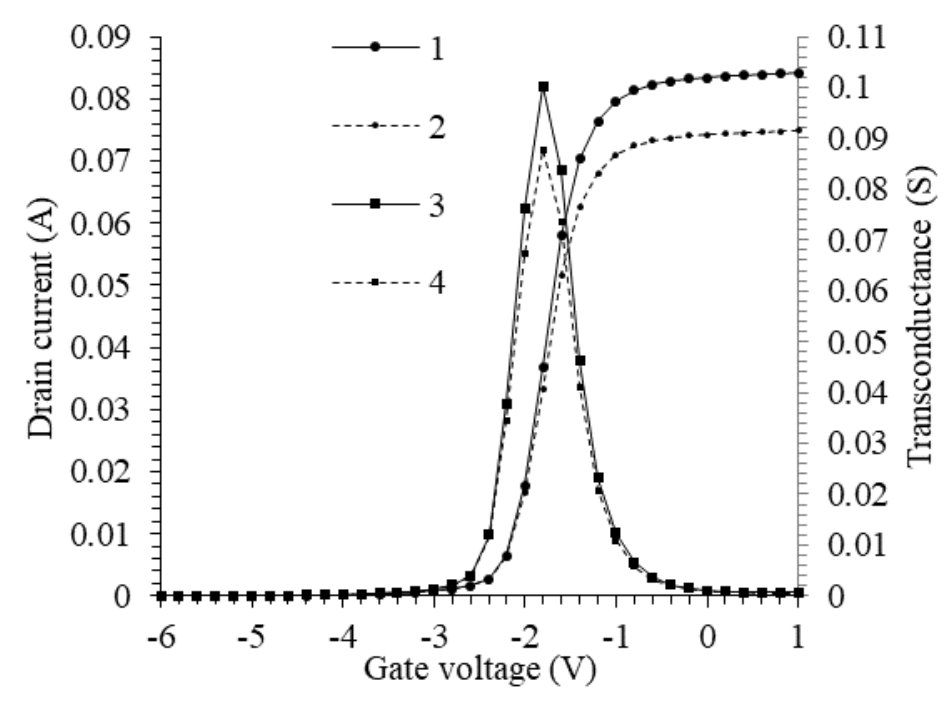

Fig. 4. Dependences of the drain current ( 1 and 2 ) and the transconductance ( 3 and 4 ) on the gate voltage at the drain voltage of $1 \mathrm{~V}: 1$ and 3 -Sotoodeh mobility model, 2 and 4 -two-dimensional electron gas mobility model.

The simulation results show that the developed model predicts a reduced drain current and worse transconductance as the electron mobility in the transistor channel is calculated to be smaller than that determined using the bulk GaAs model.

This work was supported by the grants 3.2.04 of Belarusian National Scientific Research Program "Photonics, Opto- and Microelectronics" and T18M-108 of the Belarusian Republican Foundation for Fundamental Research.

\section{References}

1. R. Dingle, H.L. Störmer, A.C. Gossard, W. Wiegmann, Appl. Phys. Lett. 33, 665 (1978)

2. T. Mimura, S. Hiyamizu, T. Fujii, K. Nanbu, Jpn. J. Appl. Phys. 19, L225 (1980)

3. W. Walukiewicz, J. Lagowski, H.C. Gatos, J. Appl. Phys. 53, 769 (1982)

4. S. Hiyamizu, J. Saito, K. Nanbu, T. Ishikawa, Jpn. J. Appl. Phys. 22, L609 (1983)

5. S. Hiyamizu, T. Fujii, T. Mimura, K. Nanbu, J. Saito, H. Hashimoto, Jpn. J. Appl. Phys. 20, L455 (1981)

6. K. Lee, M.S. Shur, T.J. Drummond, H. Morkoc, J. Appl. Phys. 54, 6432 (1983)

7. P.J. Price, Ann. Phys. 133, 217 (1981)

8. B.L. Gelmont, M. Shur, M. Stroscio, J. Appl. Phys. 77, 657 (1995)

9. Y.P. Varshni, Physica 34, 149 (1967)

10. W. Nakwaski, Physica B 201, 1 (1995)

11. O. Madelung, Semiconductors: Data Handbook (Springer, New York, 2004)

12. J.D. Zook, Phys. Rev. 136, A869 (1964)

13. M.A. Littlejohn, J.R. Hauser, T.H. Glisson, J. Appl. Phys. 48, 4587 (1977)

14. S. Adachi, Properties of Aluminum Gallium Arsenide (INSPEC, 1993)

15. M. Sotoodeh, A.H. Khalid, A.A. Rezazadeh, J. Appl. Phys. 87, 2890 (2000) 